\author{
И.В. Ракитская, И.Н. Цыба, Н.Ф. Яровая
}

ГОО ВПО «Донецкий национальный медицинский университет имени М. Горького», Донецк

\title{
ВНЕБОЛЬНИЧНАЯ ПНЕВМОНИЯ: НЕОБХОДИМЫЕ ДИАГНОСТИЧЕСКИЕ И ТЕРАПЕВТИЧЕСКИЕ ПОДХОДЫ
}

\section{О ПРЕДЕЛЕНИЕ}

Внебольничная пневмония (ВП) - пневмония, развившаяся вне стационара у пациента при отсутствии иммунодефицитных состояний либо диагностированная в первые 48 ч. с момента госпитализации [1].

\section{э П д ми ология}

Во всем мире ВП остается одной из ведущих причин смерти и сокращения продолжительности жизни [2]. Госпитализированные пациенты с ВП имеют высокую внутрибольничную смертность (около 13\%), причем исключение из статистики тяжелых больных и лиц, проживающих в домах престарелых, не приводит к существенному снижению этого показателя (2,4\% госпитализированных пациентов умирают в течение 72 ч. после поступления в стационар) [3]. Как заболеваемость, так и смертность от ВП зависят от возраста и сопутствующих заболеваний $[4,5]$. Согласно данным официальной статистики, заболеваемость ВП в РФ в 2016 г. составила 418,02 на 100 тыс. населения с ростом этого показателя по сравнению с 2015 г. на 24 \% [6].

Даже после выписки из больницы смертность от сопутствующих заболеваний, особенно у пожилых, остается высокой: согласно недавнему немецкому исследованию, в группе пациентов, средний возраст которых был больше 80 лет, большинство имели хронические неврологические или сердечно-сосудистые заболевания, смертность после выписки в течение 30 дней после госпитализации составила 4,7\% [5]. Приведенные данные послужили причиной пересмотра вопросов оценки пациентов, тактики ведения и лечения ВП. Помимо раннего установления цели лечения в отделении неотложной помощи, важным начальным этапом является быстрое выявление пациентов с высоким риском, которые нуждаются в неотложном лечении, для достижения наилучшего результата.

Основной причиной смерти больных с тяжелой ВП является рефрактерная гипоксия, септи- ческий шок и полиорганная недостаточность. В проспективных исследованиях основными факторами, ассоциированными с неблагоприятным прогнозом больных с тяжелой ВП, были: возраст старше 70 лет, проведение искусственной вентиляции легких, двусторонняя пневмония, сепсис и инфицирование P. aeroginosa [6].

Учитывая актуальность и значимость обсуждаемой проблемы, лекция должна позволить читателю:

ознакомиться с эпидемиологическими особенностями ВП, включая факторы риска и прогноз;

оценить соответствующую стратификацию риска, ведущую к обоснованным рекомендациям по лечению, как амбулаторному, так и стационарному;

знать диагностические и терапевтические шаги, которые следует предпринимать при ведении больных с ВП.

\section{ДИАГНОСТИКА}

Проявления ВП включают респираторные симптомы (кашель, мокрота, одышка, боль в груди) и общие симптомы инфекции (лихорадка, недомогание, гриппоподобные симптомы, симптомы нарушения сознания), наряду с соответствующими физикальными данными (тахипноэ, тахикардия, артериальная гипотензия, очаговая аускультативная симптоматика). Поскольку вышеперечисленная симптоматика малоспецифична, для подтверждения диагноза рекомендуется проведение рентгенологического исследования грудной клетки [7]. Инфильтраты также можно обнаружить с помощью УЗИ грудной клетки [8]. Следующие клинические данные повышают вероятность выявления инфильтрации легочной ткани при рентгенологическом исследовании грудной клетки:

- отсутствие ринореи;

(с) И.В. Ракитская, И.Н. Цыба, Н.Ф. Яровая, 2020

(c) Университетская Клиника, 2020 
- одышка;

- очаговая аускультативная симптоматика;

- лихорадка, тахикардия более 100 уд / мин;

- повышенные биомаркеры (например, C-реактивный белок).

При наличии двух и более критериев вероятность наличия инфильтрата у пациента при острой инфекции нижних дыхательных путей возрастает от менее 5\% до 18\% и более [9].

Обычно наличие инфильтрата в легочной ткани помогает в дифференциальной диагностике ВП и острого бронхита, причем антибактериальная терапия в последнем случае не требуется, так как ее нежелательные эффекты в таких ситуациях перевешивают их благотворное влияние на симптомы согласно соответствующему мета-анализу [10]. Прокальцитонин является еще одним биомаркером, который может быть использован для того, чтобы избегать ненужного назначения антибиотиков амбулаторным больным с инфекциями нижних дыхательных путей [11]. При отсутствии возможности определения этого биомаркера в месте оказания медицинской помощи можно использовать «отложенное назначение», чтобы избежать необоснованной антибиотикотерапии.

\section{СПЕКТР ПАТОГЕНОВ}

Безусловно, самый важный бактериальный патоген, вызывающий ВП, - это Streptococcus pneumoniae. Mycoplasma pneumoniae следует также учитывать в основном у пациентов в возрасте до 60 лет и без сопутствующих заболеваний. Другие патогены включают Haemophilus influenzae и, в зимний сезон, вирусы гриппа [12]. Пациенты, имеющие хронические сопутствующие заболевания и проживающие в домах престарелых, или те, у кого пневмония имеет тяжелое течение, могут быть (редко) заражены энтеробактериями (Escherichia coli, Klebsiella spp.) или Staphylococcus aureus, Legionella spp. [8, 13].

\section{АМБУЛАТОРНОЕ ЛЕЧЕНИЕ}

\section{Стратификация риска}

Основной целью стратификации риска в амбулаторных условиях является точное выявление пациентов с низким риском смерти от пневмонии и поддающихся амбулаторному лечению [8]. Впечатление врача о клинической тяжести должно быть объективизировано с помощью правильно утвержденных критериев. С этой целью рекомендуется использовать шкалу CURB65, которая легко рассчитывается без какихлибо лабораторных исследований $[14,15]$. Тем не менее у пациентов с сопутствующими заболеваниями прогноз может быть плохим, несмо- тря на низкий балл при оценке по CURB-65 [14], а острые сердечно-сосудистые осложнения у пациентов с ВП также значительно ухудшают прогноз. Больные часто нуждаются в госпитализиции из-за гипоксемии, несмотря на низкий показатель CURB-65. Недостаточное прогностическое значение критериев CURB-65 может быть заметно усилено при дополнительном рассмотрении других параметров: насыщения крови кислородом, содержания альбумина в плазме крови, определения $\mathrm{pH}$ артериальной крови (шкала SMART-COP и ее модификации) [16].

Амбулаторное лечение внебольничной пневмонии

У пациентов с умеренной ВП, которая поддается амбулаторному лечению в соответствии с критериями, изложенными выше, микробиологическое определение возбудителя обычно не требуется [17]. Рекомендуемое антибактериальное лечение для пациентов без сопутствующих заболеваний - монотерапия высокими дозами амоксициллина. Пациенты с легкой формой ВП с хроническими сопутствующими заболеваниями должны лечиться комбинацией препаратов, включающих ингибитор бета-лактамазы (амоксициллин/клавулановая кислота), который расширяет спектр эффективности для охвата S. aureus, энтеробактерий и Н. influenzae, продуцирующих бета-лактамазу [18]. Пациентам с аллергией или не переносящим пенициллин можно назначать «респираторные» фторхинолоны с эффективностью против пневмококков (моксифлоксацин, левофлоксацин). Рандомизированные исследования показали, что использование этих антибиотиков в амбулаторных условиях приводит к излечиваемости от 76 до 89\% без каких-либо существенных различий между отдельными классами веществ. Не следует назначать пероральные цефалоспорины из-за их недостаточной пероральной биодоступности, риска возникновения псевдомембранозного колита наряду с повышенным риском неудачи лечения в амбулаторных условиях. Соответствующие рекомендации по лечению приведены в таблице 1. Пациенты, получающие амбулаторное лечение, должны проходить клиническую переоценку в течение 48-72 ч., поскольку это период времени, в течение которого может наблюдаться клиническое ухудшение, несмотря на продолжающееся лечение. Когда терапия не дает улучшения, необходима госпитализация в подавляющем большинстве случаев. Если есть клиническое улучшение, включая отсрочку, то антибактериальное лечение должно продолжаться не более 5-7 дней [17]. 


\section{ЛЕЧЕНИЕ В СТАЦИОНАРЕ}

Стратификация риска в отделении неотложной помощи: внебольничная пневмония как неотложное состояние

Пациенты, нуждающиеся в ИВЛ и вазопрессорной терапии, госпитализируются в отделение интенсивной терапии. Как показано в исследовании CAPNETZ, летальность у этой группы больных возрастает до 48\% [16].

Предикторами неблагоприятного исхода являются:

тахикардия и тахипное;

гипотензия;

гипотермия;

новые нарушения сознания.

В частности, пациенты с системной гипотензией, острой дыхательной недостаточностью (ДН) или декомпенсированными сопутствующими заболеваниями сердца имеют высокий риск летальности, даже если им не требуется немедленная инфузионная терапия [19-21]. В текущих руководствах рекомендуется использовать следующие критерии (табл. 2.) как сред- ство объективизации прогнозов высокого риска тяжелых пневмоний [17]. Недавний метаанализ показал, что все девять критериев являются предикторами необходимости инфузионной терапии; если присутствует более двух критериев, чувствительность и специфичность составляют 79\% и 82\% [22]. Более того, в исследованиях реализация алгоритма лечения, включающего эти критерии (табл. 2.), понижала смертность пациентов высокого риска данного типа пневмоний с 24 до 6\% [21]. Оценка дисфункции органов вследствие сепсиса должна быть дополнена структурированной оценкой потенциально нестабильных коморбидных состояний. Среди них сердечно-сосудистые осложнения, такие как острый инфаркт миокарда или левожелудочковая недостаточность, имеют особое прогностическое значение [23].

Тактика ведения при острой дыхательной недостаточности

У пациентов с ВП и развитием острой ДН быстрое введение жидкости и немедленное начало внутривенного лечения антибиотиками широ-

Таблица 1. Эмпирическое начальное лечение легкой внебольничной пневмонии, которую можно лечить амбулаторно [17].

\begin{tabular}{lcc}
\hline \multicolumn{1}{c}{ Тяжесть течения ВП } & $\begin{array}{c}\text { Стартовая терапия } \\
\text { (стандартная доза) }\end{array}$ & $\begin{array}{c}\text { Альтернативное лечение } \\
\text { (стандартная доза) }\end{array}$ \\
\hline \hline & & Моксифлоксацин (400 мг в сутки) \\
Левофлоксацин (500 мг в сут- & ки или 2 раза в день) \\
Легкая ВП без сопутствующей па- & Амоксициллин $(750-1000$ & Кларитромицин (500 \\
тологии, амбулаторное лечение & мг три раза в день) & мг два раза в день) \\
& & Азитромицин (500 мг \\
& & в сутки $\times 3$ дня) \\
\hline
\end{tabular}

Легкая пневмония с сопутствующей патологией, амбулаторное лечение - хроническая сердечная недостаточность - неврологическое заболевание с дисфагией

Амоксициллин / клавулановая кислота (1 г три раза в день)
Моксифлоксацин (400 мг в сутки) Левофлоксацин (500 мг в сутки или 2 раза в день)

- тяжелая ХОБЛ, бронхоэктазия

- прикованный к постели пациент

Примечания: ВП - внебольничная пневмония, ХОБЛ - хроническая обструктивная болезнь легких.

Критерии прогноза высокого риска летальности внебольничной пневмонии [17]

Таблица 2.

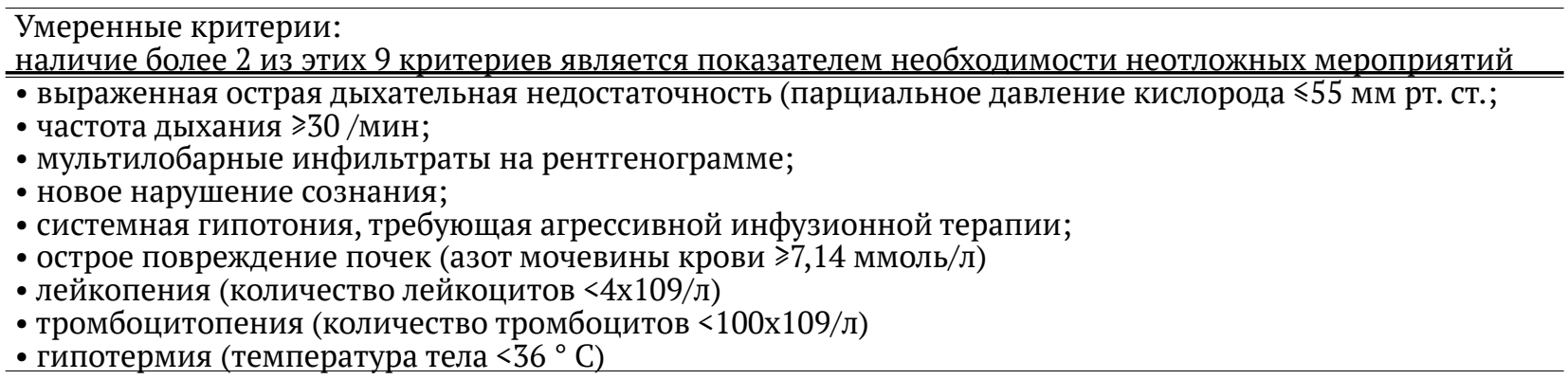


Комплекс мероприятий при тяжелой внебольничной пневмонии [17].

Комплекс мероприятий при тяжелой ВП

Должны быть выполнены как можно быстрее (в течение первых 3 ч):

- измерение сывороточного лактата;

- исследование культуры крови;

- начало соответствующего (обычно комбинированного) внутривенного лечения анти-

биотиками широкого спектра действия, в течение одного часа, если это возможно;

- у пациентов с артериальной гипотензией или повышенным уровнем лак-

тата в сыворотке быстрое внутривенное введение кристаллоидов;

- оценка (включая анализ газов крови) и лечение, при необхо-

димости, острой дыхательной недостаточности.

Должны быть выполнены в течение первых 6 ч:

- назначение вазопрессоров, если ответ на восполнение объема неадекватен;

- повторение измерения лактата, если исходно уровни были повышены;

- повторная оценка газов крови.

Примечание: ВП - внебольничная пневмония

кого спектра действия улучшают клинический исход [21]. Результаты лечения пациентов с тяжелой ВП можно улучшить при соблюдении требований соответствующих руководств по тактике ведения данной категории больных (табл. 3.) [21]. Концентрация лактата в сыворотке крови должна измеряться в приемном покое: если он повышен, необходимо быстрое проведение инфузионной терапии с повторной проверкой через короткие интервалы до нормализации его уровня. У пациентов с острой гиперкапнической ДН или сопровождающимся отеком легких следует начинать неинвазивную вентиляцию легких [17]. Недавние исследования показали, что назальная кислородная терапия с высоким расходом для пациентов с первичной гипоксической ДН, как правило, уменьшает потребность в интубации и значительно снижает смертность больных [24].

\section{Микробиологическая диагностическая оцен-} ка в стационаре

В современных рекомендациях указывается, что, как только пациент госпитализируется по поводу ВП, необходимо взять посев крови (по возможности до того, как будет дана первая доза антибиотика), в моче должны быть измерены антигены легионеллы и пневмококка, а мокрота (если имеется) должна быть исследована под микроскопом [17]. При соответствующей эпидемиологической обстановке пациентов с тяжелой ВП следует также проверять на наличие вирусов гриппа с выполнением полимеразной цепной реакции (ПЦР). При подозрении на микоплазменную пневмонию можно исследовать микоплазменный IgM или выполнить ПЦР, в то время как исследование IgG и IgA микоплазмы, серологическая диагностика хламидий не имеют диагностической ценности. При наличии плеврального выпота показана быстрая диагности- ческая пункция с последующей микробиологической оценкой и измерением $\mathrm{pH}$.

\section{ЭМПИРИЧЕСКОЕ ЛЕЧЕНИЕ АНТИБИОТИКАМИ}

У пациентов с наличием выраженной ДН эмпирическую антимикробную терапию следует начинать как можно скорее после установления диагноза, что улучшает результат; всем остальным пациентам рекомендуется начать лечение в течение 8 ч. [17]. Стартовая терапия должна проводиться парентерально в течение минимум 48 ч. в достаточно высокой дозе и охватывать пневмококки, H. influenzae, S. aureus и энтеробактерии. Препаратом выбора является внутривенный бета-лактамный антибиотик (табл. 4.). Даже у пациентов с почечной недостаточностью рекомендуемая максимальная доза должна назначаться как минимум в течение первых 24 ч. Для пациентов с ВП и органной недостаточностью дополнительно рекомендуется введение макролида в качестве стартовой терапии ввиду его влияния на легионеллу, что улучшает исход (абсолютное снижение смертности с 24 до 21\% [25]. При наличии клинического ответа и отсутствии атипичных патогенов макролид может быть отменен через 3 дня лечения. Для госпитализированных пациентов без органной недостаточности дополнительный препарат группы макролидов не обязателен, так как не продемонстрированы улучшения в проспективных плацебо-контролируемых клинических исследованиях [26].

Pseudomonas aeruginosa вызывает ВП крайне редко (менее 1\%). Факторы риска включают тяжелую хроническую обструктивную болезнь легких, бронхоэктатическую болезнь или постоянную чрескожную эндоскопическую гастростомическую трубку. Препаратом выбора являет- 
Эмпирическое стартовое стационарное лечение внебольничной пневмонии [17].

\begin{tabular}{|c|c|c|}
\hline Тяжесть течения ВП & $\begin{array}{l}\text { Стартовая терапия } \\
\text { (стандартная доза) }\end{array}$ & $\begin{array}{c}\text { Альтернативное лечение } \\
\text { (стандартная доза) }\end{array}$ \\
\hline $\begin{array}{l}\text { Умеренно тяжелая ВП } \\
\text { (нет острой органной } \\
\text { недостаточности) }\end{array}$ & $\begin{array}{l}\text { Бета-лактам в/в: } \\
\text { - амоксициллин / клавулано- } \\
\text { вая кислота (2,2 г каждые } 8 \text { ч) } \\
\text { - ампициллин / сульбактам } \\
\text { (3 г каждые } 8 \text { ч) } \\
\text { - цефуроксим (1,5 г каждые } 8 \text { ч) } \\
\text { - цефтриаксон (2 г в сутки) } \\
\text { - цефотаксим (2 г, каждые } 8 \text { ч) } \\
\text { + Необязательно * макролид в/в или } \\
\text { пероральный в течение } 3 \text { дней: } \\
\text { - кларитромицин (500 мг каждые } 12 \text { ч) } \\
\text { - азитромицин (500 мг в сутки) }\end{array}$ & $\begin{array}{l}\text { «еспираторный» фторхи- } \\
\text { нолон в/в или per os: } \\
\text { Моксифлоксацин (400 мг в сутки) } \\
\text { Левофлоксацин (500 мг в сутки } \\
\text { или каждые } 12 \text { ч) }\end{array}$ \\
\hline $\begin{array}{l}\text { Тяжелая ВП } \\
\text { (острая органная } \\
\text { недостаточность) }\end{array}$ & $\begin{array}{l}\text { Бета-лактам в/в } \\
\text { - пиперациллин / тазобак- } \\
\text { там (4,5 г каждые 6-8 ч) } \\
\text { - цефтриаксон (2 г в сутки) } \\
\text { - цефотаксим (2 г каждые 6-8 ч) } \\
\text { + Макролид в/в на } 3 \text { дня } \\
\text { - кларитромицин (500 мг каждые } 12 \text { ч) } \\
\text { - азитромицин (500 мг в сутки) }\end{array}$ & $\begin{array}{l}\text { Респираторный фторхинолон в/в: } \\
\text { Моксифлоксацин ( } 400 \text { мг в сутки) } \\
\text { Левофлоксацин (500 мг каждые } 12 \text { ч) } \\
\text { (без монотерапии у пациентов } \\
\text { с септическим шоком) }\end{array}$ \\
\hline
\end{tabular}

ся пиперациллин/тазобактам, ципрофлоксацин, левофлоксацин [6]. Возможность наличия мультирезистентной микрофлоры должна быть рассмотрена у пациентов с индивидуальными факторами риска, такими как:

- предварительная госпитализация (в этом случае применяются рекомендации по нозокомиальной пневмонии);

- предварительное длительное лечение антибиотиками широкого спектра действия;

- предшествующие госпитализации поездки в страны, в которых распространены мультирезистентные микроорганизмы. Препаратом выбора являются ванкомицин, линезолид [6].

Дополнительное эмпирическое противовирусное лечение осельтамивиром следует рассмотреть для госпитализированных пациентов с острой органной недостаточностью и повышенным риском (сопутствующие заболевания, беременность) во время сезона гриппа. Осельтамивир следует отменить при получении отрицательного результата вирусного теста ПЦР. Рекомендации по стартовой эмпирической терапии пациентов, получающих лечение в стационаре, приведены в таблице 4.

\section{КЛИНИЧЕСКАЯ СТАБИЛИЗАЦИЯ И НЕУДАЧИ ЛЕЧЕНИЯ}

Все пациенты, которые соответствуют критериям тяжелой ВП или имеют сопутствующие заболевания, нуждаются в постоянном мони- торинге течения заболевания до клинического улучшения. Наибольший риск ухудшения - в течение первых 72 ч. после поступления в стационар. Для всех госпитализированных пациентов переоценка критериев стабильности должна проводится один раз в день [17]. Признаками клинической стабильности считаются:

- ЧСС $\leqslant 100$ / мин;

- частота дыхания $\leqslant 24$ / мин;

- систолическое артериальное давление $\geqslant 90$ мм рт. ст.;

- температура тела $\leqslant 37,8^{\circ} \mathrm{C}$;

- возможность принимать пищу через рот;

- нормальное состояние сознания;

- отсутствие гипоксемии (р О $2 \geqslant 60$ мм рт. ст., SaO $2 \geqslant 90 \%$ ).

В случае, когда все эти критерии соблюдены, риск осложнений течения заболевания невелик. Кроме того, рекомендуется повторное измерение СРБ или прокальцитонина через 3-4 дня; если значения не снижаются, необходим пересмотр эффективности проводимого лечения.

Если стабилизация состояния не наступает или через 3-5 дней соответствующего лечения наблюдается клиническое ухудшение, констатируется неэффективность лечения. Важно дифференцировать простую задержку в достижении стабилизации состояния, с одной стороны, и клинически прогрессирующую пневмонию с другой. Прогрессирующая пневмония имеет плохой прогноз с увеличением смертности в 10 
раз. Таким образом, клиническое прогрессирование требует немедленной диагностики, включающей переоценку критериев тяжести и функционирования органов, отслеживание параметров воспаления и исключение таких осложнений, как абсцесс или плевральный выпот. Для стабилизации состояния показан перевод пациента в отделение интенсивной терапии. Кроме того, показана повторная микробиологическая оценка, в том числе бронхоскопия, а также дифференциальная диагностика внелегочных инфекционных и неинфекционных состояний и осложнений, таких как тромбоэмболия легочной артерии или до сих пор нераспознанное состояние с подавленным иммунитетом (включая ВИЧ-инфекцию). Лечение антибиотиками у пациентов с прогрессирующей инфекцией должно покрывать любые пробелы в антимикробном спектре исходного лечения и всегда назначается в виде внутривенной комбинированной терапии в адекватных дозах.

\section{ПРОДОЛЖИТЕЛЬНОСТ Ь ЛЕЧЕНИЯ АНТИБИОТИКАМИ}

Как только критерии клинической стабильности будут достигнуты, можно перейти на пероральное введение препарата с хорошей биодоступностью (например, амоксициллин/клавулановая кислота). Лечение должно быть закончено через 2-3 дня после достижения стабилизации состояния. Таким образом, 10-14 дней лечения обычно достаточно даже для тяжелой ВП [27].

Основные этапы ведения пациента с ВП включают стратификацию риска и применение стратегии управления, адаптированной к степени тяжести заболевания, наряду с созданием структурированной последующей помощи и вторичной профилактики, особенно для пациентов с сопутствующими заболеваниями.

\section{И.В. Ракитская, И.Н. Цыба, Н.Ф. Яровая}

ГОО ВПО «Донецкий национальный медицинский университет имени М. Горького», Донецк

\section{ВНЕБОЛЬНИЧНАЯ ПНЕВМОНИЯ: НЕОБХОДИМЫЕ ДИАГНОСТИЧЕСКИЕ И ТЕРАПЕВТИЧЕСКИЕ ПОДХОДЫ}

В лекции представлены современные данные о распространенности и особенностях этиологии, факторах риска внебольничной пневмонии, показан вклад каждого из возможных возбудителей в этиологическую структуру заболевания. Приведены диагностические критерии пневмонии. Описаны основные алгоритмы оценки тяжести пневмонии и прогноза заболевания с применением различных шкал, целью которых является определение места лечения: амбулаторно или в стационаре, в отделении общего профиля или реанимации. Представлены современные рекомендации по эмпирическому выбору антибактериальных препаратов, указаны среднетерапевтические дозы антибактериальных препара- тов для лечения внебольничной пневмонии у пациентов с нормальной функцией почек. Описана тактика ведения пациентов при развитии острой дыхательной недостаточности. Рассмотрены вопросы оптимальной длительности лечения пневмонии в зависимости от этиологического фактора, представлены критерии достаточности антибактериальной терапии. Описаны причины возможной неэффективности проводимой эмпирической антибактериальной терапии внебольничной пневмонии.

Ключевые слова: внебольничная пневмония, амбулаторное лечение, лечение в стационаре, эмпирическая антибактериальная терапия, критерии достаточности антибактериальной терапии.

\section{I.V. Rakytskaya, I.N. Tsyba, N.F. Yarovaya}

SEI HPE «M. Gorky Donetsk National Medical University», Donetsk

\section{COMMUNITY-ACQUIRED PNEUMONIA:}

\section{NECESSARY DIAGNOSTIC AND THERAPEUTIC APPROACHES}

The lecture presents modern data on the prevalence and characteristics of etiology, risk factors for community-acquired pneumonia and shows the contribution of each of the possible pathogenic organisms to the etiological disease structure. Diagnostic criteria for pneumonia are given. The main algorithms for assessing the severity of pneumonia and prognosis of the disease using various scales are described, the purpose of which is to deter- mine the place of treatment: on an outpatient basis or in a hospital, in a general department or an intensive care unit. Modern recommendations on the empirical choice of antibacterial drugs are presented, the average therapeutic doses of antibacterial drugs for the treatment of community-acquired pneumonia in patients with normal kidney function are indicated. The tactics for the development of acute respiratory failure are described. The is- 
sues of the optimal pneumonia treatment duration depending on the etiology, the criteria for the adequacy of antibacterial therapy are presented. The reasons for the possible inefficiency of the empirical antibacterial therapy of community-acquired pneumonia are described.
Key words: community-acquired pneumonia, outpatient treatment, inpatient treatment, empirical antibacterial therapy, criteria for the adequacy of antibacterial therapy.

\section{ЛИТЕРАТУРА}

1. Wiersinga W.J., Bonten M.J., Boersma W.G., Jonkers R.E., Aleva R.M., Kullberg B.J., et al. Management of community-acquired pneumonia in adults: 2016 guideline update from the Dutch Working Party on Antibiotic Policy (SWAB) and Dutch Association of Chest Physicians (NVALT). Neth J Med. 2018; Jan 76(1):4-13.

2. Wunderink R.G., Waterer G.W. Clinical practice. Community-acquired pneumonia. N Engl J Med. 2014; Feb 6;370(6):543-551. doi: 10.1056/NEJMcp1214869.

3. Kolditz M., Ewig S. Community-Acquired Pneumonia in Adults. Dtsch Arztebl Int. 2017 Dec 8;114(49):838-848. doi: 10.3238/arztebl.2017.0838

4. Круглякова Л. В., Нарышкина С. В., Одиреев А. Н. Современные аспекты внебольничной пневмонии Бюллетень физиологии и патологии дыхания. 2019; 71: 120-134. DOI: 10.12737/article_5c89acc410e1f3.79881136

5. Kolditz M., Tesch F., Mocke L., Höffken G., Ewig S., Schmitt J. Burden and risk factors of ambulatory or hospitalized CAP: A population based cohort study. Respir Med. 2016; Dec;121:32-38. doi: 10.1016/j.rmed.2016.10.015

6. Клинические рекомендации: внебольничная пневмония (проект). Российское респираторное общество; Межрегиональная ассоциация по клинической микробиологии и антимикробной химиотерапии. 2018. 88 с. URL: pulmo.ru/obrazovatelnye-resursy/federalnyeklinicheskie-rekomendatsii/ (дата обращения: 07.12.2019).

7. Marchello C.S., Ebell M.H., Dale A.P., Harvill E.T., Shen Y., Whalen C.C. Signs and symptoms that rule out community-acquired pneumonia in outpatient adults: a systematic review and meta-analysis. J Am Board Fam Med. 2019; MarApr;32(2):234-247. doi: 10.3122/jabfm.2019.02.180219

8. Froes F, Pereira JG,Póvoa P.Outpatient management of community-acquired pneumonia. Curr Opin Infect Dis. 2018; Apr;31(2):170-176. doi: 10.1097/QCO.0000000000000435

9. Minnaard M.C., de Groot J.A.H., Hopstaken R.M., Schierenberg A., de Wit N.J., Reitsma J.B. The added value of C-reactive protein measurement in diagnosing pneumonia in primary care: a meta-analysis of individual patient data. CMAJ. 2017; Jan 16;189(2):E56-E63. doi: 10.1503/cmaj. 151163

10. Smith S.M., Fahey T., Smucny J., Becker L.A. Antibiotics for acute bronchitis. Cochrane Database Syst Rev. 2017; Jun 19;6:CD000245. doi: 10.1002/14651858.CD000245. pub4

11. Schuetz P., Wirz Y., Sager R., Christ-Crain M., Stolz D., Tamm M., et al. Procalcitonin to initiate or discontinue antibiotics in acute respiratory tract infections. Cochrane Database Syst Rev. 2017; Oct 12;10:CD007498. doi: 10.1002/14651858.CD007498.pub3

12. Chen J., Li X., Wang W., Jia Y., Lin F., Xu J. The prevalence of respiratory pathogens in adults with community-acquired pneumonia in an outpatient cohort. Infect Drug Resist. 2019; Jul 30;12:2335-2341. doi: 10.2147/IDR.S213296

13. Pletz M.W., Rohde G., Schütte H., Bals R., von Baum H., Welte T. Epidemiology and Aetiology of Community-acquired Pneumonia (CAP). Dtsch Med Wochenschr. 2011; Apr;136(15):775-80. doi: 10.1055/s-0031-1275806

14. Ewig S., Bauer T., Richter K., Szenscenyi J., Heller G., Strauss R., et al. Prediction of in-hospital death from community-acquired pneumonia by varying CRBage groups. Eur Respir J. 2013; Apr;41(4):917-22. doi: $10.1183 / 09031936.00065212$

15. Ebell MH. Community-acquired pneumonia: determining safe treatment in the outpatient setting. Am Fam Phy-

\section{REFERENCES}

1. Wiersinga W.J., Bonten M.J., Boersma W.G., Jonkers R.E., Aleva R.M., Kullberg B.J., et al. Management of community-acquired pneumonia in adults: 2016 guideline update from the Dutch Working Party on Antibiotic Policy (SWAB) and Dutch Association of Chest Physicians (NVALT). Neth J Med. 2018; Jan 76(1):4-13.

2. Wunderink R.G., Waterer G.W. Clinical practice. Community-acquired pneumonia. N Engl J Med. 2014; Feb 6;370(6):543-551. doi: 10.1056/NEJMcp1214869

3. Kolditz M., Ewig S. Community-Acquired Pneumonia in Adults. Dtsch Arztebl Int. 2017; Dec 8;114(49):838-848. doi: 10.3238/arztebl.2017.0838

4. Kruglyakova L. V., Naryshkina S. V., Odireev A. N. Sovremennye aspekty vnebol'nichnoi pnevmonii Byulleten' fiziologii i patologii dykhaniya. 2019; 71: 120-134. DOI: 10.12737/article 5c89acc410e1f3.79881136 (in Russian)

5. Kolditz M., Tesch F., Mocke L., Höffken G., Ewig S., Schmitt J. Burden and risk factors of ambulatory or hospitalized CAP: A population based cohort study. Respir Med. 2016; Dec;121:32-38. doi: 10.1016/j.rmed.2016.10.015

6. Klinicheskie rekomendatsii: vnebol'nichnaya pnevmoniya (proekt). Rossiiskoe respiratornoe obshchestvo; Mezhregional'naya assotsiatsiya po klinicheskoi mikrobiologii i antimikrobnoi khimioterapii. 2018. 88 s. URL: pulmo.ru/obrazovatelnye-resursy/federalnyeklinicheskie-rekomendatsii/ (data obrashcheniya: 07.12.2019) (in Russian).

7. Marchello C.S., Ebell M.H., Dale A.P., Harvill E.T., Shen Y., Whalen C.C. Signs and symptoms that rule out community-acquired pneumonia in outpatient adults: a systematic review and meta-analysis. J Am Board Fam Med. 2019; MarApr;32(2):234-247. doi: 10.3122/jabfm.2019.02.180219

8. FroesF,Pereira JG,Póvoa P.Outpatient management of community-acquired pneumonia. Curr Opin Infect Dis. 2018; Apr;31(2):170-176. doi: 10.1097/OCO.0000000000000435

9. Minnaard M.C., de Groot J.A.H., Hopstaken R.M., Schierenberg A., de Wit N.J., Reitsma J.B. The added value of C-reactive protein measurement in diagnosing pneumonia in primary care: a meta-analysis of individual patient data. CMAJ. 2017; Jan 16;189(2):E56-E63. doi: $10.1503 / \mathrm{cm}$ aj. 151163

10. Smith S.M., Fahey T., Smucny J., Becker L.A. Antibiotics for acute bronchitis. Cochrane Database Syst Rev. 2017; Jun 19;6:CD000245. doi: 10.1002/14651858.CD000245. pub4

11. Schuetz P., Wirz Y., Sager R., Christ-Crain M., Stolz D., Tamm M., et al. Procalcitonin to initiate or discontinue antibiotics in acute respiratory tract infections. Cochrane Database Syst Rev. 2017; Oct 12;10:CD007498. doi: 10.1002/14651858.CD007498.pub3

12. Chen J., Li X., Wang W., Jia Y., Lin F., Xu J. The prevalence of respiratory pathogens in adults with community-acquired pneumonia in an outpatient cohort. Infect Drug Resist. 2019; Jul 30;12:2335-2341. doi: 10.2147/IDR.S213296

13. Pletz M.W., Rohde G., Schütte H., Bals R., von Baum H., Welte T. Epidemiology and Aetiology of Community-acquired Pneumonia (CAP). Dtsch Med Wochenschr. 2011; Apr;136(15):775-80. doi: 10.1055/s-0031-1275806

14. Ewig S., Bauer T., Richter K., Szenscenyi J., Heller G., Strauss R., et al. Prediction of in-hospital death from community-acquired pneumonia by varying CRBage groups. Eur Respir J. 2013; Apr;41(4):917-22. doi: $10.1183 / 09031936.00065212$

15. Ebell MH. Community-acquired pneumonia: determin- 
sician. 2019; Jun 15:99(12):768-769. PMID:31194484

16. Kolditz M., Ewig S., Schütte H., Suttorp N., Welte T., Rohde G. Assessment of oxygenation and comorbidities improves outcome prediction in patients with community-acquired pneumonia with a low CRB-65 score. J Intern Med. 2015; Aug;278(2):193-202. doi: 10.1111/joim.12349

17. Ewig S., Höffken G., Kern W.V., Rohde G., Flick H., Krause R. Management of Adult Community-acquired Pneumonia and Prevention - Update 2016. Pneumologie. 2016; Mar;70(3):151-200. doi: 10.1055/s-0042-101873

18. Tomczyk S., Jain S., Bramley A.M., Self W.H., Anderson E.J., Trabue C., et al. Antibiotic Prescribing for Adults Hospitalized in the Etiology of Pneumonia in the Community Study. Open Forum Infect Dis. 2017; Jun 20;4(2):ofx088. doi: $10.1093 /$ ofid/ofx088

19. Jain S., Self W.H., Wunderink R.G., Fakhran S., Balk R., Bramley A.M., et al. Community-Acquired Pneumonia Requiring Hospitalization among U.S. Adults. N Engl J Med. 2015; Jul 30;373(5):415-27. doi: 10.1056/NEJMoa1500245

20. Ferrer R., Martin-Loeches I., Phillips G., Osborn T.M., Townsend S., Dellinger R.P., et al. Empiric antibiotic treatment reduces mortality in severe sepsis and septic shock from the first hour: results from a guideline-based performance improvement program. Crit Care Med. 2014; Aug;42(8):1749-55. doi: 10.1097/CCM.0000000000000330

21. Lim H.F., Phua J., Mukhopadhyay A., Ngerng W.J., Chew M.Y., Sim T.B., et al. IDSA/ATS minor criteria aid pre-intensive care unit resuscitation in severe community-acquired pneumonia Eur Respir J. 2014; Mar;43(3):852-62. doi: $10.1183 / 09031936.00081713$

22. Salih W., Schembri S., Chalmers J. D. Simplification of the IDSA/ATS criteria for severe CAP using meta-analysis and observational data. Eur Respir J. 2014; Mar;43(3):842-51. doi: $10.1183 / 09031936.00089513$

23. Corrales-Medina V.F., Musher D.M., Wells G.A., Chirinos J.A., Chen L., Fine M.J. Cardiac complications in patients with community-acquired pneumonia: incidence, timing, risk factors, and association with short-term mortality. Circulation. 2012; Feb 14;125(6):773-81. doi: 10.1161/ CIRCULATIONAHA.111.040766

24. Coudroy R., Frat J.P., Ehrmann S., Pène F., Terzi N., Decavèle M., et al. High-flow nasal oxygen therapy alone or with non-invasive ventilation in immunocompromised patients admitted to ICU for acute hypoxemic respiratory failure: the randomised multicentre controlled FLORALIIM protocol. BMJ Open. 2019; Aug 10;9(8):e029798. doi: 10.1136/bmjopen-2019-029798

25. Sligl W.I., Asadi L., Eurich D.T., Tjosvold L., Marrie T.J., Majumdar S.R. Macrolides and mortality in critically ill patients with community-acquired pneumonia: a systematic review and meta-analysis. Crit Care Med. 2014; Feb;42(2):420-32. doi: 10.1097/CCM.0b013e3182a66b9b

26. Cremers A.J., Sprong T., Schouten J.A., Walraven G., Hermans P.W., Meis J.F., et al. Effect of antibiotic streamlining on patient outcome in pneumococcal bacteraemia. J Antimicrob Chemother. 2014; Aug;69(8):2258-64. doi: 10.1093/jac/dku109

27. Uranga A., España P.P., Bilbao A., Quintana J.M., Arriaga I., Intxausti M., et al. Duration of Antibiotic Treatment in Community-Acquired Pneumonia: A Multicenter Randomized Clinical Trial. JAMA Intern Med. 2016; Sep 1;176(9):1257-65. doi: 10.1001/jamainternmed.2016.3633 ing safe treatment in the outpatient setting. Am Fam Physician. 2019; Jun 15;99(12):768-769.

16. Kolditz M., Ewig S., Schütte H., Suttorp N., Welte T., Rohde G. Assessment of oxygenation and comorbidities improves outcome prediction in patients with community-acquired pneumonia with a low CRB-65 score. I Intern Med. 2015; Aug;278(2):193-202. doi: 10.1111/joim.12349

17. Ewig S., Höffken G., Kern W.V., Rohde G., Flick H., Krause R. Management of Adult Community-acquired Pneumonia and Prevention - Update 2016. Pneumologie. 2016; Mar;70(3):151-200. doi: 10.1055/s-0042-101873

18. Tomczyk S., Jain S., Bramley A.M., Self W.H., Anderson E.J., Trabue C., et al. Antibiotic Prescribing for Adults Hospitalized in the Etiology of Pneumonia in the Community Study. Open Forum Infect Dis. 2017; Jun 20;4(2):ofx088. doi: 10.1093/ofid/ofx088

19. Jain S., Self W.H., Wunderink R.G., Fakhran S., Balk R., Bramley A.M., et al. Community-Acquired Pneumonia Requiring Hospitalization among U.S. Adults. N Engl J Med. 2015; Jul 30;373(5):415-27. doi: 10.1056/NEJMoa1500245

20. Ferrer R., Martin-Loeches I., Phillips G., Osborn T.M., Townsend S., Dellinger R.P., et al. Empiric antibiotic treatment reduces mortality in severe sepsis and septic shock from the first hour: results from a guideline-based performance improvement program. Crit Care Med. 2014; Aug;42(8):1749-55. doi: 10.1097/ CCM.0000000000000330.

21. Lim H.F., Phua J., Mukhopadhyay A., Ngerng W.J., Chew M.Y., Sim T.B., et al. IDSA/ATS minor criteria aid pre-intensive care unit resuscitation in severe community-acquired pneumonia Eur Respir J. 2014; Mar;43(3):852-62. doi: 10.1183/09031936.00081713

22. Salih W., Schembri S., Chalmers J. D. Simplification of the IDSA/ATS criteria for severe CAP using meta-analysis and observational data. Eur Respir J. 2014; Mar;43(3):842-51. doi: 10.1183/09031936.00089513

23. Corrales-Medina V.F., Musher D.M., Wells G.A., Chirinos J.A., Chen L., Fine M.J. Cardiac complications in patients with community-acquired pneumonia: incidence, timing, risk factors, and association with short-term mortality. Circulation. 2012; Feb 14;125(6):773-81. doi: 10.1161/ CIRCULATIONAHA.111.040766

24. Coudroy R., Frat J.P., Ehrmann S., Pène F., Terzi N., Decavèle M., et al. High-flow nasal oxygen therapy alone or with non-invasive ventilation in immunocompromised patients admitted to ICU for acute hypoxemic respiratory failure: the randomised multicentre controlled FLORALIIM protocol. BMJ Open. 2019; Aug 10;9(8):e029798. doi: 10.1136/bmjopen-2019-029798

25. Sligl W.I., Asadi L., Eurich D.T., Tjosvold L., Marrie T.J., Majumdar S.R. Macrolides and mortality in critically ill patients with community-acquired pneumonia: a systematic review and meta-analysis. Crit Care Med. 2014; Feb;42(2):420-32. doi: 10.1097/CCM.0b013e3182a66b9b

26. Cremers A.J., Sprong T., Schouten J.A., Walraven G., Hermans P.W., Meis J.F., et al. Effect of antibiotic streamlining on patient outcome in pneumococcal bacteraemia. J Antimicrob Chemother. 2014; Aug;69(8):2258-64. doi: 10.1093/jac/dku109

27. Uranga A., España P.P., Bilbao A., Quintana J.M., Arriaga I., Intxausti M., et al. Duration of Antibiotic Treatment in Community-Acquired Pneumonia: A Multicenter Randomized Clinical Trial. JAMA Intern Med. 2016; Sep 1;176(9):1257-65. doi: 10.1001/jamainternmed.2016.3633 